\title{
Angewandte
}

Supporting Information

(c) Wiley-VCH 2006

69451 Weinheim, Germany 


\section{Ultrastable Particle-Stabilized Foams}

Urs T. Gonzenbach, André R. Studart *, Elena Tervoort, Ludwig J. Gauckler

$\left.{ }^{\star}{ }^{\star}\right]$ M.Sc. U.T. Gonzenbach, Dr. A.R. Studart, Dr. E. Tervoort, Prof. Dr. L.J.Gauckler Department of Materials

ETH Zurich

Wolfgang-Pauli-Strasse 10, HCI G 539

Zurich, CH 8093, Switzerland

Fax: +41-44-632-1132

E-mail:andre.studart@mat.ethz.ch

\section{Experimental Section}

The colloidal particles used in this study were acquired from the following suppliers: $\delta-\mathrm{Al}_{2} \mathrm{O}_{3}, d_{50} \sim 70 \mathrm{~nm}$, Nanophase Technologies Co. (Romeoville, IL, USA); $\alpha-\mathrm{Al}_{2} \mathrm{O}_{3}, d_{50} \sim 200 \mathrm{~nm}$ (grade Ceralox HPA-0.5), Sasol North America Inc. (Tucson, AZ, USA); $\alpha-\mathrm{Al}_{2} \mathrm{O}_{3}, d_{50} \sim 2000 \mathrm{~nm}$ (grade CL 2500 SG), Alcoa Co. (Ludwigshafen, Germany); $\mathrm{ZrO}_{2}, d_{50} \sim 50 \mathrm{~nm}$ (grade TZ-3Y-E), Tosoh Corporation (Tokyo, Japan); $\beta-\mathrm{Ca}_{3} \mathrm{PO}_{4}, d_{50} \sim 50 \mathrm{~nm}$, Flame Powders AG (Schlieren, Switzerland); $\mathrm{SiO}_{2}, d_{50} \sim 80 \mathrm{~nm}$ (grade Snowtex ZL), Nissan Chemical (Houston, TX, USA). All other chemicals were purchased from Fluka AG (Buchs, Switzerland). Prior to foaming, colloidal suspensions were prepared by adding the dry powders to the liquid solvent upon steady mixing. The suspension solids concentration varied between 15 and 45 vol\%, depending on the powder, solvent and short amphiphiles 
used. Surface lyophobization of initially hydrophilic particles was carried out by first de-agglomerating the powder through a standard ball-milling process, followed by the gradual addition of aqueous solutions containing the short amphiphiles. Concentrated $2 \mathrm{M} \mathrm{HCl}$ or $1 \mathrm{M} \mathrm{NaOH}$ solutions (Titrisol, Fluka AG, Buchs, Switzerland) were used for $\mathrm{pH}$ adjustments. The adsorption of amphiphiles on the particle surface was indirectly determined by measuring the concentration of amphiphiles left in the bulk liquid media after $2 \mathrm{~h}$ mixing of the powder with the amphiphile. The bulk concentration was determined by removing the particles in a two-step centrifugation process (Z513K, Hermle Labortechnik GmbH, 4,500 rpm, 1h; 5417R, Eppendorf AG, 15,000 rpm, $20 \mathrm{~min}$ ) and subsequently measuring the amphiphilic concentration in the supernatant solutions by potentiometric titration (DT-1200, Dispersion Technology, Inc., Mount Kisco, NY, USA). The suspension surface tension was assessed with the pendant drop method (PAT1, Sinterface Technologies $\mathrm{GmbH}$, Berlin, Germany) ${ }^{[1]}$, using $15-35 \mathrm{~mm}^{3}$ suspension droplets pending in air. Foams were produced by thoroughly mixing the concentrated suspensions with a planetary mixer (Major Classic, Kenwood Ltd, Havant, UK) for 3 minutes at full speed. The whipped cream and egg white foams used for comparison were also prepared by mechanical frothing for 3 minutes. The shaving foam (Gillette Foam, Regular, Gillette Co, London) was taken directly from the product container and was not submitted to any further processing. The foamability was measured from the weight and volume of the foam obtained. Transmitted light microscopy (Polyvar MET, Reichert-Jung, Austria) was used to investigate foam stability by monitoring the bubble size distribution of wet foams over time. Fluorescent silica particles and hexyl amine was used for the confocal laser scanning microscopy images. The labeled silica particles $\left(d_{50} \sim 500 \mathrm{~nm}\right)$ were synthesized following the procedure described in reference ${ }^{[2]}$ and consisted of a 
silica core ( $400 \mathrm{~nm})$, a fluorescent layer around the core $(\sim 10 \mathrm{~nm})$ and an outer silica rim ( $100 \mathrm{~nm})$. Concentrated silica foams (15 vol\%) were prepared with a hand-mixer at $\mathrm{pH} 10.2$ with $30 \mathrm{mM}$ of hexyl amine. Hollow colloidosomes were obtained by diluting $(20 x)$ the concentrated foams and were subsequently harvested for imaging in the confocal microscope.

\section{Foams with different materials}

Particle-stabilized foams with several different materials were prepared using the method described in the article. Table S1 depicts the exact conditions required for surface lyophobization of various types of colloidal particles and the resulting features of the foams produced by mechanical shearing. Foams exhibiting high air content and small mean bubble sizes were achieved by appropriately selecting the anchoring group and tail length of the amphiphile molecule according to the surface chemistry of the colloidal particles used (Table S1).

\section{Role of free amphiphiles:}

The reduction in surface tension required for foaming was shown to be due to the combined effect of modified particles and non-adsorbed free amphiphiles (see Figure 3 of the article). However, it is important to note that even though the non-adsorbed amphiphiles also decrease the suspension surface tension (Figure 3), no stable foams were obtained upon mechanical shearing of aqueous solutions containing solely the amphiphilic molecules. Bubbles created during mixing 
promptly disappeared when mechanical shearing was stopped. Additional foaming experiments were carried out to confirm that the foams obtained do not result from the adsorption of free amphiphiles at the air-water interface in combination with an increase of the viscosity of the foam lamella due to the presence of particles. These experiments were performed with suspensions containing the amphiphile butyl amine and a high concentration of alumina particles (35 vol\%) in water at $\mathrm{pH} 4.75$. Since amines do not adsorb at the alumina surface at acidic $\mathrm{pHs}$, the amphiphiles present in this suspension are free to adsorb at the air-water interface, while the completely hydrophilic alumina particles remain within the suspension liquid medium. No stable foam was produced by mechanical shearing these aminecontaining alumina suspensions, confirming the major role of the interfaceadsorbed lyophobized particles on the preparation of highly-stable foams.

\section{Stabilization mechanism:}

Among the several mechanisms leading to foam destabilization ${ }^{[3]}$, bubble disproportionation had so far been particularly difficult to avoid in liquid foams due to the ever-present difference in Laplace pressure between bubbles of distinct sizes, which ultimately results in a steady diffusion of gas molecules from smaller to larger bubbles over time. ${ }^{[3]}$ The remarkable resistance of our particle-stabilized foams against coalescence and disproportionation is most likely imparted by the strong attachment of particles at the air-water interface (Figure 4 in the article) and by the formation of an attractive particle network at the interface and throughout the foam lamella. ${ }^{[4,5]}$ 
Particles attached to the air-water interface can reduce the overall foam free energy by thousands of $k T s$, if a considerable amount of interfacial area is replaced upon adsorption. ${ }^{[6,7]}$ Such a reduction in free energy makes the interfacial adsorption of partially lyophobic particles an irreversible process, as opposed to the continuous adsorption and desorption of conventional surfactant molecules at the air-water interface (Gibbs-Marangoni effect). Particles strongly adsorbed at the interface may resist the shrinkage of small bubbles during disproportionation by forming a percolating interfacial armor that mechanically withstands the low pressures resulting from gas diffusion outwards the bubble. ${ }^{[8]}$ The fact that the air bubbles are highly confined throughout the foam volume may also contribute to the enhanced stability by restricting the movement of particles attached to the interface. In this case, the immobile attached particles would hinder the mobility of the airwater interface in a similar way to the well-known pinning effect of particles in grain boundaries of polycrystalline materials. This effect arises when a moving interface is constrained by an interface-adsorbed particle due to local equilibrium along the triple-phase junction. ${ }^{[9]}$

The attractive network of particles formed at the air-water interface and within the foam lamella is also expected to withstand the overpressure created in larger bubbles and prevent their expansion during disproportionation. The attractive particle network was formed either due to the screening effect of the adsorbed amphiphile (in the case of carboxylates and amines) or due to the high concentration of counter-ions in solution (in the case of gallates). Such conditions lead to a decrease of the electrical double layer thickness around particles, favoring their coagulation by van der Waals and hydrophobic attractive forces. The formation of such attractive colloidal network in particle-stabilized foams was confirmed by confocal microcopy images of concentrated foams. The fact that the foam stability 
was enhanced by the addition of screening salt ions strongly indicates the important role of this attractive network. ${ }^{[5]}$

\section{Comparison with flotation additives:}

The lyophobization approach used in our method resembles that applied for the separation of micron- to millimeter-sized ore particles in flotation processes. However, the hydrophobic tail of the amphiphiles used in the flotation of oxides is considerably longer (typically $>10$ carbons) than that of the short-chain molecules used in this work. ${ }^{[10-14]}$ Shorter molecules such as xanthates exhibiting usually 3 to 5 carbons are also used in flotation, but have been predominantly applied for the separation of electrically conductive sulfide ores ${ }^{[15]}$ and are usually inefficient in the recovery of oxide particles. ${ }^{[16]}$ It should also be emphasized that the flotation process relies on the phase separation (creaming) of bubbles in order to purify the mineral ore, as opposed to our approach where no creaming effect is desired. 


\section{REFERENCES}

[1] D. Mobius, R. Miller, in Studies in Interface Science, Vol. 11 (Eds.: D. Mobius, R. Miller), Elsevier, Amsterdam, 2001, p. 521.

[2] A. Vanblaaderen, A. Vrij, Langmuir 1992, 8, 2921.

[3] A. J. Wilson, in Springer Series in Applied Biology (Ed.: A. W. Robards), Springer-Verlag, Berlin, 1989, p. 233.

[4] Z. P. Du, M. P. Bilbao-Montoya, B. P. Binks, E. Dickinson, R. Ettelaie, B. S. Murray, Langmuir 2003, 19, 3106.

[5] E. Dickinson, R. Ettelaie, T. Kostakis, B. S. Murray, Langmuir 2004, 20, 8517.

[6] B. P. Binks, Current Opinion in Colloid \& Interface Science 2002, 7, 21.

[7] R. Aveyard, B. P. Binks, J. H. Clint, Advances in Colloid and Interface Science 2003, 100, 503.

[8] S. I. Kam, W. R. Rossen, Journal of Colloid and Interface Science 1999, 213, 329.

[9] A. P. Sutton, R. W. Balluffi, in Monographs on the Physics and Chemistry of Materials (Eds.: R. J. Brook, A. Cheetham, A. Heuer, P. Hirsch, T. J. Marks, J. Silcox, D. A. Smith, M. V. Tirrell, V. Vitek), Oxford University Press, Oxford, 2003, p. 820.

[10] B. M. Moudgil, P. K. Singh, J. J. Adler, in Handbook of Applied Surface and Colloid Chemistry, Vol. 1 (Ed.: K. Holmberg), John Wiley \& Sons Ltd., West Sussex, 2002, p. 591.

[11] J. Shibata, D. W. Fuerstenau, International Journal of Mineral Processing 2003, 72, 25.

[12] T. W. Healy, P. Somasundaran, D. W. Fuerstenau, International Journal of Mineral Processing 2003, 72, 3.

[13] D. W. Fuerstenau, M. Colic, Colloids and Surfaces a-Physicochemical and Engineering Aspects 1999, 146, 33.

[14] S. C. Lu, S. X. Song, Colloids and Surfaces 1991, 57, 49.

[15] R. Wood, in Principles of Mineral Processing (Eds.: M. H. Jones, J. T. Woodcock), Australian Institute of Mining and Metallurgy, 1984, p. 91-115.

[16] J. D. Miller, J. Li, J. C. Davidtz, F. Vos, Minerals Engineering 2005, 18, 855. 


\section{FIGURE AND TABLE CAPTIONS}

Table S1 Particle-stabilized foams produced from a variety of metal oxide colloidal particles using the approaches outlined in Figure 1 of the article.

Figure S1: Outstanding stability of particle-stabilized foams in comparison to stateof-the-art food and cosmetic foams. No drainage, creaming and disproportionation was observed in all particle-stabilized foams prepared with partially lyophobic particles (exemplified in $d$ for alumina and valeric acid), as compared to the considerable destabilization that takes place in well-established cosmetic (a, shaving foam) and food foams (b, whipped cream; c, egg white foam). Images shown on the left-hand side were taken 5 minutes after foaming, whereas those on the right-hand side were taken after 4, 69, 67 and 100 hours for the shaving (a), whipped cream (b), egg white (c) and particle-stabilized foams (d), respectively (scale bar: $50 \mu \mathrm{m}$ ). Due to their remarkable stability, particle-stabilized foams can be used to produce bulk macroporous materials $\left(>500 \mathrm{~cm}^{3}\right)$ upon drying and sintering, as exemplified by the inset image in (d) for alumina (scale bar: $50 \mu \mathrm{m}$ ). 
TABLE

Table S1: Particle-stabilized foams produced from a variety of metal oxide colloidal particles using the approaches outlined in Figure 1 of the article.

\begin{tabular}{|c|c|c|c|c|c|c|c|}
\hline \multicolumn{2}{|c|}{ Particle } & \multicolumn{2}{|c|}{ Amphiphile } & \multirow{2}{*}{ Solvent } & \multirow{2}{*}{$\begin{array}{l}\text { Solids } \\
\text { content } \\
\text { (vol\%) }\end{array}$} & \multirow{2}{*}{$\begin{array}{l}\text { Foamability } \\
\text { (\% air) }\end{array}$} & \multirow{2}{*}{$\begin{array}{c}\text { Mean } \\
\text { bubble } \\
\text { size }(\mu \mathrm{m})\end{array}$} \\
\hline Type & $\begin{array}{c}\text { Size, } d_{50} \\
(n m)\end{array}$ & Type & $\begin{array}{l}\text { Conc. } \\
\text { (mol/L) }\end{array}$ & & & & \\
\hline$\delta-\mathrm{Al}_{2} \mathrm{O}_{3}$ & 70 & \multirow{4}{*}{$\begin{array}{l}\text { Valeric } \\
\text { acid }\end{array}$} & 0.011 & \multirow{4}{*}{$\begin{array}{c}\text { Water, pH } \\
4.75\end{array}$} & 5 & 88 & 130 \\
\hline$\delta-\mathrm{Al}_{2} \mathrm{O}_{3}$ & 70 & & 0.050 & & 20 & 90 & 47 \\
\hline$\alpha-\mathrm{Al}_{2} \mathrm{O}_{3}$ & 200 & & 0.030 & & 35 & 80 & 26 \\
\hline$\alpha-\mathrm{Al}_{2} \mathrm{O}_{3}$ & 2000 & & 0.020 & & 25 & 66 & 40 \\
\hline$\alpha-\mathrm{Al}_{2} \mathrm{O}_{3}$ & 200 & \multirow{2}{*}{$\begin{array}{l}\text { Propyl } \\
\text { gallate }\end{array}$} & 0.100 & $\begin{array}{c}\text { Water, pH } \\
9.9\end{array}$ & 35 & 83 & 30 \\
\hline $\mathrm{ZrO}_{2}$ & 50 & & 0.080 & $\begin{array}{c}\text { Water, pH } \\
9.9\end{array}$ & 22 & 79 & 70 \\
\hline $\mathrm{Ca}_{3} \mathrm{PO}_{4}$ & 50 & $\begin{array}{c}\text { Butyl } \\
\text { gallate }\end{array}$ & 0.040 & $\begin{array}{c}\text { Water, pH } \\
9.9\end{array}$ & 17 & 86 & 45 \\
\hline $\mathrm{SiO}_{2}$ & 80 & $\begin{array}{l}\text { Hexyl } \\
\text { amine }\end{array}$ & 0.065 & $\begin{array}{c}\text { Water, pH } \\
10.6\end{array}$ & 35 & 82 & 30 \\
\hline
\end{tabular}


FIGURES

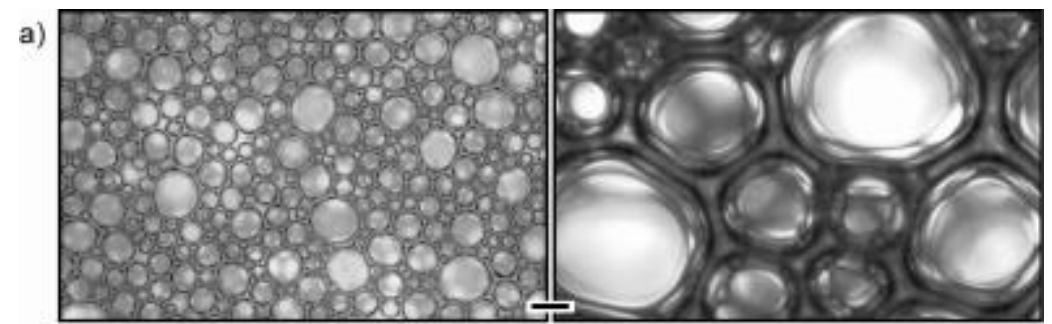

b)

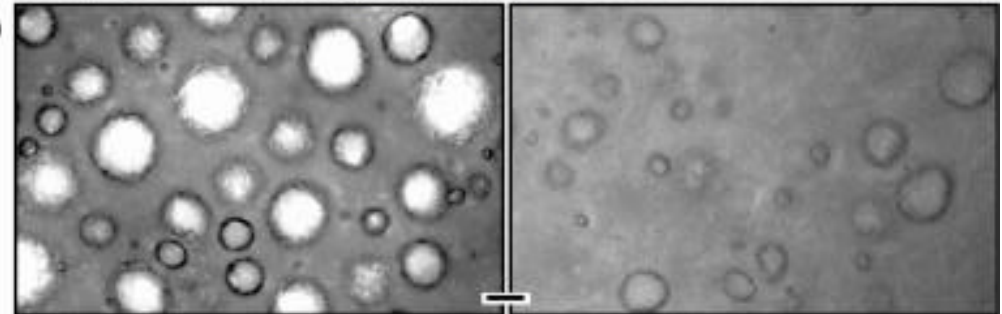

c)

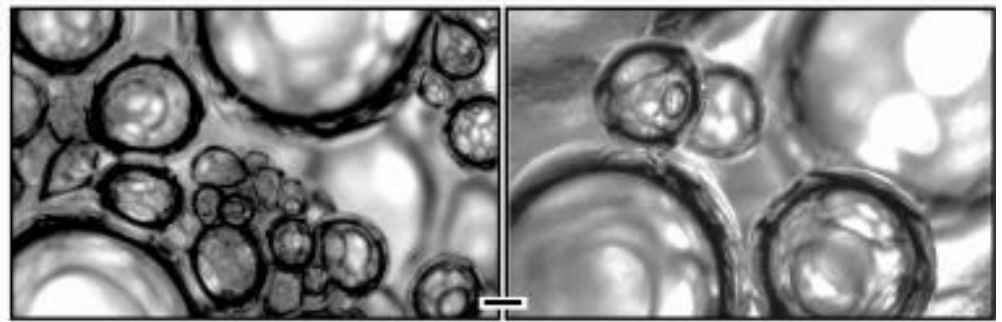

d)

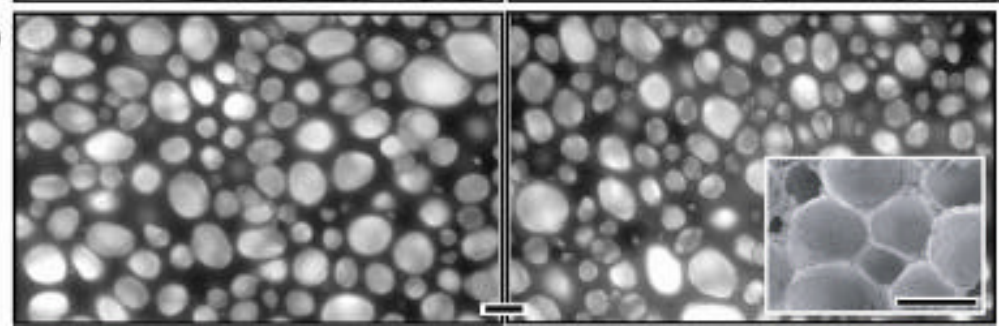

Figure S1 